\title{
Selection footprints reflect genomic changes associated with breeding efforts in 56 cucumber inbred lines
}

Bin Liu (1)', Dailu Guan $\mathbb{E}^{2}$, Xuling Zhai ${ }^{1}$, Sen Yang ${ }^{1}$, Shudan Xue ${ }^{1}$, Shuying Chen ${ }^{1}$, Jing Huang ${ }^{3}$, Huazhong Ren ${ }^{1}$ and Xingwang Liu'

\begin{abstract}
Cucumber selective breeding over recent decades has dramatically increased productivity and quality, but the genomic characterizations and changes associated with this breeding history remain unclear. Here, we analyzed the genome resequencing data of 56 artificially selected cucumber inbred lines that exhibit various phenotypes to detect trait-associated sequence variations that reflect breeding improvement. We found that the 56 cucumber lines could be assigned to group 1 and group 2, and the two groups formed a distinctive genetic structure due to the breeding history involving hybridization and selection. Differentially selected regions were identified between group 1 and group 2, with implications for genomic-selection breeding signatures. These regions included known quantitative trait loci or genes that were reported to be associated with agronomic traits. Our results advance knowledge of cucumber genomics, and the 56 selected inbred lines could be good germplasm resources for breeding.
\end{abstract}

\section{Introduction}

Cucumber (Cucumis sativus L.; $2 n=2 x=14$ ) is generally considered to be an economically important vegetable crop worldwide ${ }^{1}$, as well as a well-characterized model for studying fleshy fruit development ${ }^{2}$. Cultivated cucumber varieties have evolved from their wild progenitors under natural and artificial selection ${ }^{3,4}$. The domestication of cucumber began $\sim 3000$ years ago, and since then, different breeding strategies have been used to produce desired characteristics in order to meet the demand for human nutrition and health ${ }^{5}$. However, it was reported that cucumber had a low species diversity due to narrow bottlenecks ${ }^{3}$; therefore, the breeding of new cucumber varieties that require low inputs and are

\footnotetext{
Correspondence: Huazhong Ren (renhuazhong@cau.edu.cn) or

Xingwang Liu (Liuxw01@cau.edu.cn)

${ }^{1}$ Beijing Key Laboratory of Growth and Developmental Regulation for Protected Vegetable Crops, College of Horticulture, China Agricultural University, Beijing 100193, P. R. China

${ }^{2}$ Centre for Research in Agricultural Genomics (CRAG), CSIC-IRTA-UAB-UB, Campus Universitat Autònoma de Barcelona, Bellaterra 08193, Spain Full list of author information is available at the end of the article.
}

environmentally sustainable will probably still be challenging in the future.

Traditionally, the breeding of cucumber germplasm was accomplished simply by selecting lines with desirable characteristics for propagation, while the new methodology has accompanied the development of high-volume parallel genotyping and sequencing technologies, for instance, genomic selection ${ }^{6}$. However, selective cucumber breeding employing genomic markers was initiated only in the $1980 \mathrm{~s}^{5,7-9}$, and the implementation of genomic selection in cucumber breeding is still difficult. Although artificial selection has greatly increased cucumber productivity and quality with expected improvements in traits, such as gynoecy, disease resistance, uniform ripening and bitterness ${ }^{10-13}$, genetic gains in genomic selection are pending. Next-generation DNA sequencing technologies now allow cost-effective genome sequencing at a population scale, which has led to the construction of variation maps for crop plants such as maize ${ }^{14}$, rice ${ }^{15}$, soybean $^{16}$, sorghum ${ }^{17}$, apple ${ }^{18}$, watermelon ${ }^{19}$, pepper ${ }^{20}$, and cucumber ${ }^{3}$. Meanwhile, genome-wide association

\section{(c) The Author(s) 2019}

(c) (i) Open Access This article is licensed under a Creative Commons Attribution 4.0 International License, which permits use, sharing, adaptation, distribution and reproduction cc) in any medium or format, as long as you give appropriate credit to the original author(s) and the source, provide a link to the Creative Commons license, and indicate if changes were made. The images or other third party material in this article are included in the article's Creative Commons license, unless indicated otherwise in a credit line to the material. If material is not included in the article's Creative Commons license and your intended use is not permitted by statutory regulation or exceeds the permitted use, you will need to obtain permission directly from the copyright holder. To view a copy of this license, visit http://creativecommons.org/licenses/by/4.0/. 
studies (GWASs) are used to detect genetic variations that underlie many important and complex traits in plants ${ }^{21}$. To date, some loci or genes have been identified by genomic studies in cucumber with large natural populations $^{3,22,23}$. However, very few studies have identified loci or genomic regions in populations exposed to long-term artificial selection.

In recent decades, we focused on breeding elite cucumbers with outstanding phenotypes by crossing excellent Asian and European germplasm and performing artificial selection. On the one hand, we aimed to combine desirable agronomic traits from Asian and Eurasian lines in the progenies. On the other hand, we always selected the seeds from healthy plants and removed the disease-infected plants; therefore, most of the lines performed well in the field and were free of disease infection. As a result of long-term selection, we obtained 56 elite cucumber lines that retained phenotypic diversity. In this case, we would like to know the genomic characterizations and changes associated with this breeding history and the potential of our breeding materials.

Here, we resequenced the 56 cucumber lines and found that their genetic background was significantly different from that previously reported for 115 cucumber lines ${ }^{3}$. The data from our 56 lines revealed specific selected regions related to breeding efforts, and these selected regions were associated with the agronomic performance of cucumber varieties and harbored many reportedly important genes.

\section{Results and Discussion}

\section{Determination of cucumber genetic structure with artificially selected inbred lines}

The genetic data of 56 lines subjected to artificial selection represent both genomic variations and their usefulness in cucumber breeding improvement. According to our breeding aims, the 56 lines were assigned to group 1 (G1), which has a background similar to that of East Asian (EA) cucumber lines, and group 2 (G2), which has the Eurasian (EU) background (Supplementary Table 1; Supplementary Fig. 1). These lines were selected to obtain fruit traits with commercial value, such as normal vs abnormal fruit shoulder (Fig. 1 a1), dull vs glossy fruit $\operatorname{skin}^{24}$ (Fig. 1 a2), uniform vs nonuniform fruit color $^{12}$ (Fig. 1 a3), green vs yellow-green fruit color (Fig. 1 a4), trichome and tubercule presence vs trichome and tubercule absence $^{25,26}$ (Fig. 1 a5-6), large vs small tubercules ${ }^{27}$ (Fig. 1 a7, a7-B a7-S), and hard vs soft spines ${ }^{28}$ (Fig. 1 a8, a7-H a8-S). To generate the 56 lines, at least 13 founder lines from the EA or EU market were selected for crossfertilization. Next, backcrossing or selfing was performed to obtain recombinant inbred lines or near-isogenic lines with the target traits.
We also included 115 lines sequenced by Qi et al. that were publicly available from the National Center for Biotechnology Information (NCBI, https://www.ncbi.nlm. nih.gov/ $)^{3}$. Resequencing of the 56 selected lines generated a total of 1.6 billion paired-end reads, with an average depth of $\sim 18 \times$ and coverage of $98.4 \%$ (Supplementary Table 1). By aligning paired-end reads against the reference genome of the inbred cucumber line $9930^{1}$, a total of $\sim 8.4$ million single nucleotide polymorphism (SNPs) were identified (Fig. 1b). Among these SNPs, 67187 were unique in our breeding lines, indicating that the artificial selection lines were enriched with cucumber genomic variations, which are likely important for cucumber breeding.

To examine the genetic structure of the 56 inbred lines, principal component analysis (PCA) ${ }^{29}$ and ADMIXTURE analysis $^{30}$ were employed. It was clear that our cucumber lines formed groups different from the previously published EA, EU, Indian domesticated (IND), Indian wild (INW) and Xishuangbanna (XSBN) groups (Fig. 1c, d, Supplementary Figs. 2 and 3). Along the first component, explaining $31 \%$ of the variation, our 56 selected lines clustered away from the others (Fig. 1c), which was supported by ADMIXTURE analysis when $K=2$ (Fig. 1d). Within the 56 lines, separation was found between the two groups along the second component in the PCA and at $K=6$ in the ADMIXTURE analysis (Fig. 1c, d). When the $K$-value with the lowest cross-validation error $(K=7)$ was used, the G1, G2, EA, EU, IND, INW, and XSBN groups were assigned to independent clusters (Fig. 1d). However, the EA cluster was divided into two different genetic backgrounds, which was somewhat different from the pattern observed with published data ${ }^{3}$ (Fig. 1d). In addition, this kind of clustering was also reinforced in the constructed neighbor-joining (NJ) tree ${ }^{31}$ (Supplementary Fig. 2), indicating that our 56 cucumber lines had a different genetic structure than the 115 lines $^{3}$ obtained by selective breeding and suggesting that G1 and G2 represent ideal populations for cucumber breeding.

\section{Evidence of introgression and genetic diversity in two groups of cucumber}

Using the TreeMix ${ }^{32}$ model and the seven populations, we detected that G1 and G2 were phylogenetically similar to the EA population, and the migration edge signaled introgression of EU into G1 and G2, especially to G2 (Fig. 2a), when admixture was modeled to include two migrations, suggesting that EU cucumbers were the donors used to improve G1 and G2 by breeding. This result perfectly matched our selection schemes (Supplementary Table 1). When admixture was modeled to include five migrations, we detected that migration edges to EA, G1, and G2 lines were from XSBN. Surprisingly, we did not find any introgression from INW lines (Supplementary Fig. 4). 


\section{a}
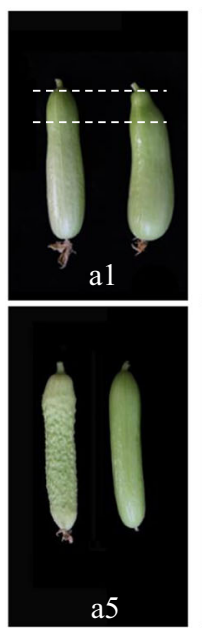

b
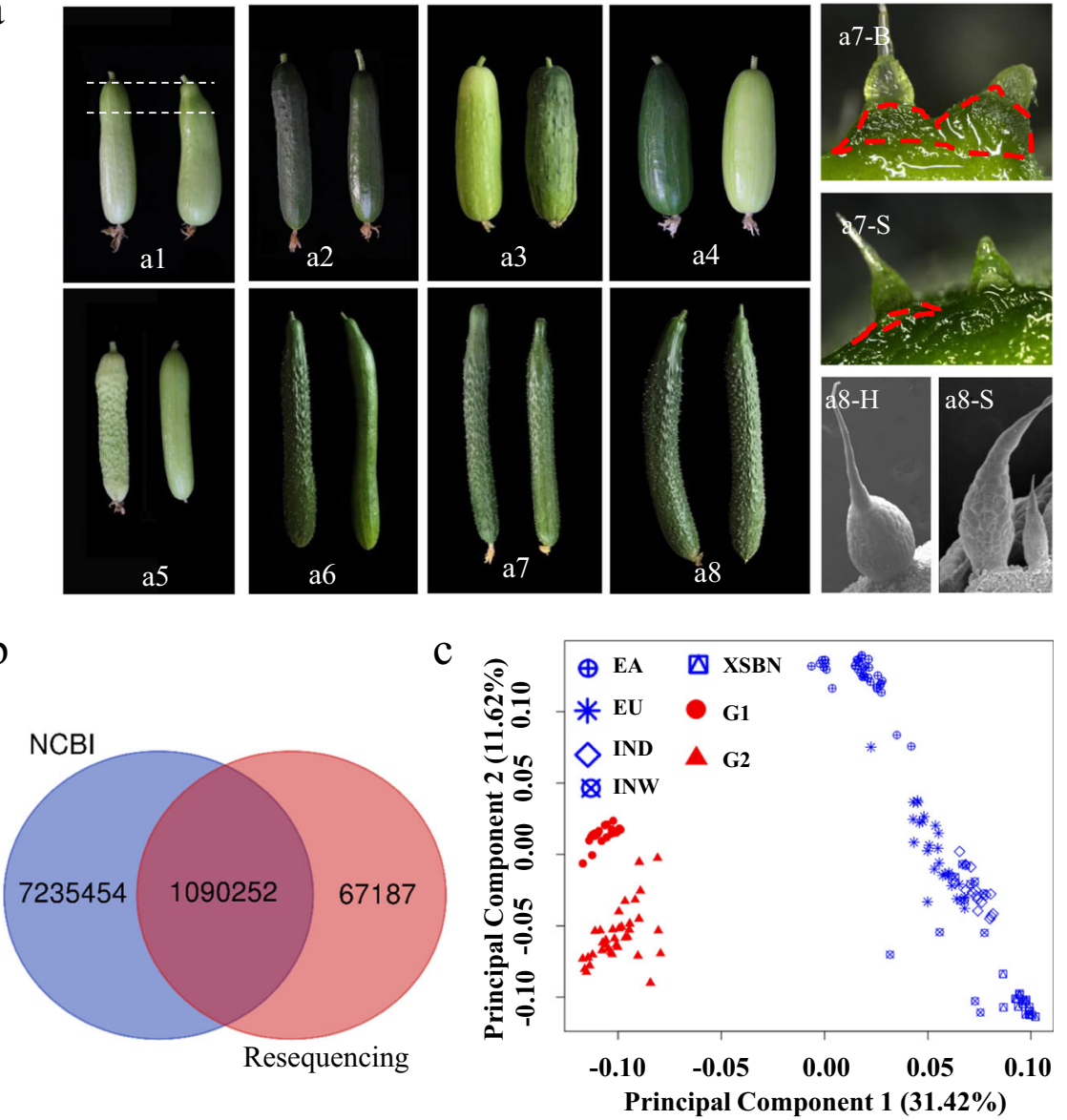

d
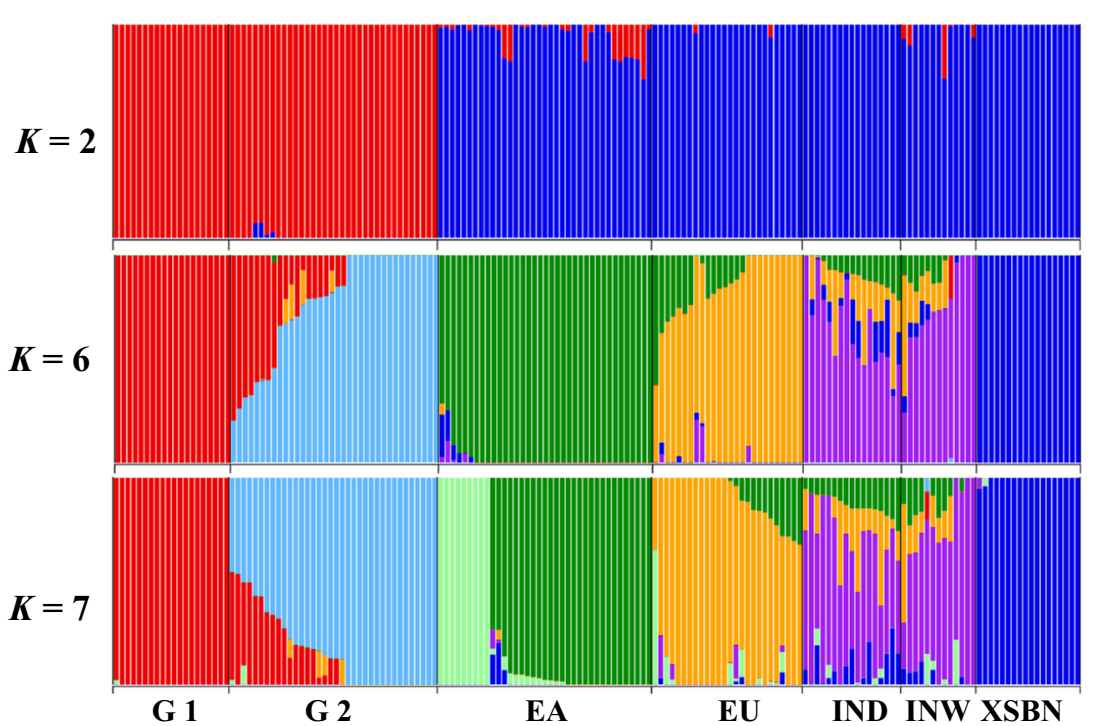
(see figure on previous page)

Fig. 1 Determination of cucumber genetic structure and genomic variations in $\mathbf{5 6}$ artificial selection inbred lines. a Representatives of 56 cucumber lines with different phenotypes: (a1) Normal vs abnormal fruit shoulder (the part between the two white dotted lines). (a2) Dull vs glossy skin. (a3) Uniform vs nonuniform fruit color. (a4) Green vs yellow-green skin. (a5) Trichome and tubercule presence vs trichome and tubercule absence in yellow-green-skinned fruit. (a6) Trichome and tubercule presence vs trichome and tubercule absence in green-skinned fruit. (a7) Large tubercules (a7-B) vs small tubercules (a7-S). The tubercules are marked by red dotted lines. (a8) Hard spines (a8-H) vs soft spines (a8-S). b Venn diagram depicting unique and shared SNPs between the 56 resequenced lines and 115 previously reported lines. c Principal component analysis (PCA) of the 171 cucumber lines. The PCA considered principal components 1 (PC1) and 2 (PC2), which explained 31.42\% and 11.62\% of the variance, respectively. $\mathbf{d}$ ADMIXTURE analysis ( $K=2,6$, and 7). Each bar represents one individual, and the length of the colored bar represents the proportion of the cucumber genome inherited from each ancestral population. East Asian (EA); Eurasian (EU); Indian domesticated (IND); Indian wild (INW); Xishuangbanna (XSBN); group 1 (G1); group 2 (G2)

These signals of genetic introgression were supported by D-statistic analyses (ABBA-BABA tests) ${ }^{33}$ (Supplementary Table 2). These results suggested that G1 and G2 have experienced gene flow from other cucumber populations, which coincided with our breeding history in which G1 was improved based on the EA background and G2 was selected to change fruit traits mainly by crossing with the EU group. We also examined the residuals of the model fit to identify the relationships that were not captured by the maximum likelihood trees; the XSBN and EA groups stood out (Supplementary Fig. 5).

Increased linkage disequilibrium (LD) and reduced nucleotide diversity are expected in artificial selection lines $^{34}$. To understand the specific LD block patterns in our 56 bred cucumbers, the LD decay $\left(r^{2}\right)$ with increasing physical distance between SNPs was calculated by combining the 115 publicly available lines. The results indicated that both G1 and G2 cucumbers exhibited higher LD than previously reported for the 115 lines (Fig. 2b), suggesting that large introgressions were selected and fixed in G1 and G2. We then evaluated nucleotide diversity at the whole-genome level within different groups. The average values of genome-wide nucleotide diversity $(\pi)$ for the G1, G2, EA, EU, IND, INW, and XSBN lines were $1.56 \times 10^{-3}, 1.71 \times 10^{-3}, 1.37 \times 10^{-3}$, $2.26 \times 10^{-3}, 4.25 \times 10^{-3}, 9.27 \times 10^{-3}$, and $1.37 \times 10^{-3}$, respectively (Fig. 2c). The $\pi$ values of the INW and IND groups were higher than those of the other groups, consistent with previously published $\mathrm{data}^{22}$, while those of both G1 and G2 were significantly lower ( $t$-test, $P<2 \times$ $\left.10^{-16}\right)$. The high LD and low $\pi$ values confirmed that G1 and G2 are highly artificially selected populations.

Resequencing the 56 artificially selected cucumber lines also provided opportunities to investigate selection footprints reflecting the complex genomic and genetic variations resulting from cucumber breeding. LD and nucleotide diversity analysis demonstrated that G1 and G2 were exposed to higher selection pressure, while CLR analysis revealed clear differentiation via selection between the two groups and multiple targets of selection in G1 and G2. In addition, we found that the EU group was most likely the founder cultivar for $G 1$ and G2, especially for $\mathrm{G} 2$. In addition, it is worth mentioning that migration edges to EA, G1, and G2 lines were from XSBN (Supplementary Fig. 4). This can be explained by the fact that XSBN is a semiwild landrace with some unique traits that are very useful for cucumber breeding ${ }^{35,36}$. However, wild species of Cucumis are important as potential genetic sources for breeding ${ }^{37}$; INW is the origin population of cucumber and thus harbors higher genomic diversity than XSBN (Fig. 2c) and can be used for breeding improvement in the future.

\section{Selection regions in the two groups of cucumber}

To assess the extent of genetic differentiation in G1 and G2, we used a method to calculate pairwise differences in allele frequency $\left(F_{\mathrm{ST}}\right)$ between $\mathrm{G1}$ and $\mathrm{G} 2$, as well as the composite likelihood ratio $(\mathrm{CLR})^{38}$ to identify genomic regions differentially selected within each group. The $F_{\mathrm{ST}}$ value highlighted 89 signature sites that were distributed on chromosomes 1-6 and were significantly selected between G1 and G2 $(q<0.05$; Fig. 2d; Supplementary Table 3). However, it is intriguing that the CLR statistics indicated selection signals over the threshold (top 1\%) mainly on chromosome 1 in G1 (Fig. 3a; Supplementary Table 3) and on chromosomes 2, 3, and 5 in G2 (Fig. 3b; Supplementary Table 3). These results suggested that different target regions were under selection between the two groups during the breeding process and were consistent with different selection pressures leading to distinct groups. The selected regions of G1 and G2 detected by merging $F_{\mathrm{ST}}$ and CLR statistics contained 4074 and 3508 genes, respectively (Supplementary Table 4). Gene Ontology (GO) analysis of these genes showed enrichment in binding processes, metabolic processes and catalytic activity (Supplementary Table 5).

Previous studies identified several domestication-related genes or QTLs in cucumber, including those for disease resistance $^{39,40}$, flowering time ${ }^{41}$, fruit length ${ }^{42}$, sex determination $^{42}$, fruit bitterness ${ }^{43}$, and trichome development ${ }^{28}$. These reported genes or QTLs overlapped with the putative selective sweep regions detected in our study, indicating that a subset of domestication loci might have undergone selection for continuous improvement of 
$\mathrm{a}$

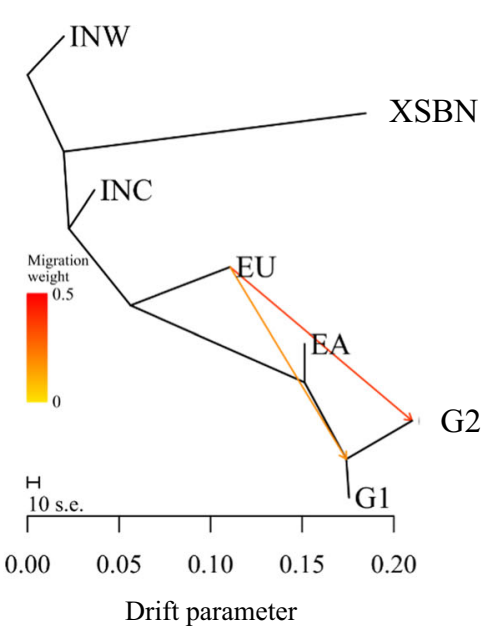

b

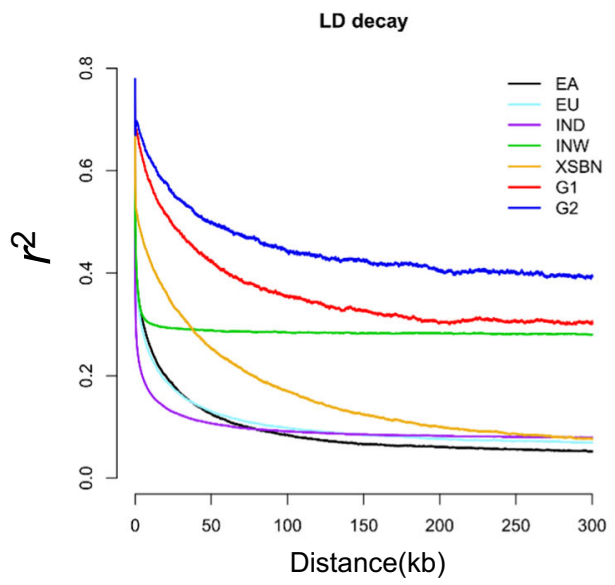

C
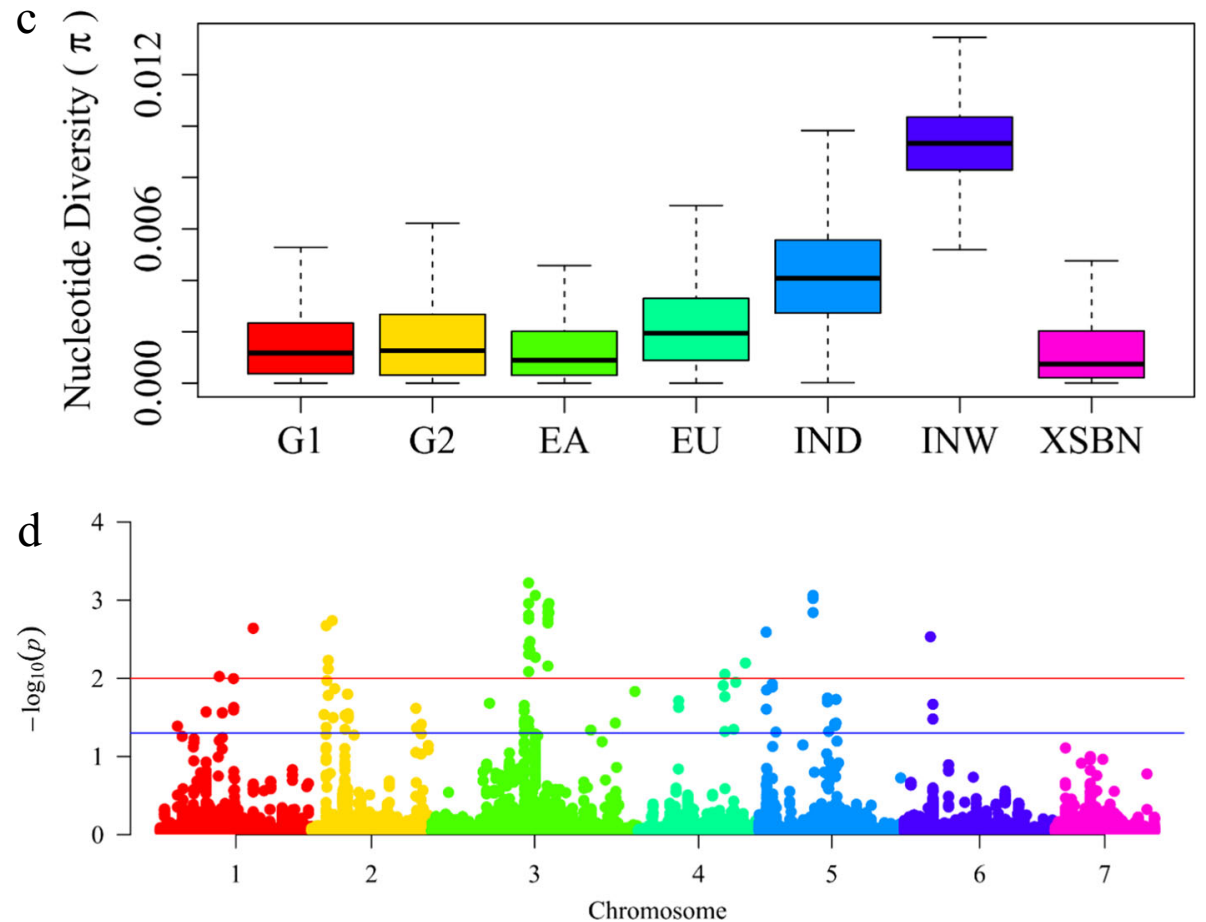

Fig. 2 Evidence of introgression and genetic diversity in two groups of cucumber. a TreeMix analysis of the inferred relationships among seven cucumber groups. Migration edges are colored according to percent ancestry received from the donor population. Scale bar shows 10 times the average standard error of the estimated entries in the sample covariance matrix. $\mathbf{b}$ LD decay of seven groups of cucumber measured by $r^{2}$ implemented in PopLDdecay software ${ }^{55}$. c Nucleotide diversity of seven groups of cucumber measured by the $\pi$ value, taking into account a $50 \mathrm{~kb}$ window and $5 \mathrm{~kb}$ sliding steps. d Population divergence $\left(F_{\mathrm{ST}}\right)$ in the 56 artificial inbred lines

important agronomic traits. For example, in the G1 population, we selected a soft trichome phenotype (Fig. 1 a8-S). As a result, Csa1G056960, which is involved in soft spine formation, was detected in the putative selected region on chromosome $1^{28}$ (Supplementary Table 4). Another selected trait in G1 was flowering time; Csa1G651710, which is responsible for flowering ${ }^{41}$, was also detected in the putative selected region on chromosome 1 (Supplementary Table 4). In G2, a bitterness trait was strongly selected, and two transcription factors, $B l$ and $B t$, on chromosome 5 that regulate the pathway of biosynthesis of cucurbitacin $C$ were detected in the selection region (Supplementary Table 4). It is known that selection imposed on the $B t$ gene during domestication led to the derivation of nonbitter cucurbits from their bitter ancestors ${ }^{43}$; therefore, our results proved that the target region for selection can be explained by the selection schemes applied. 
a

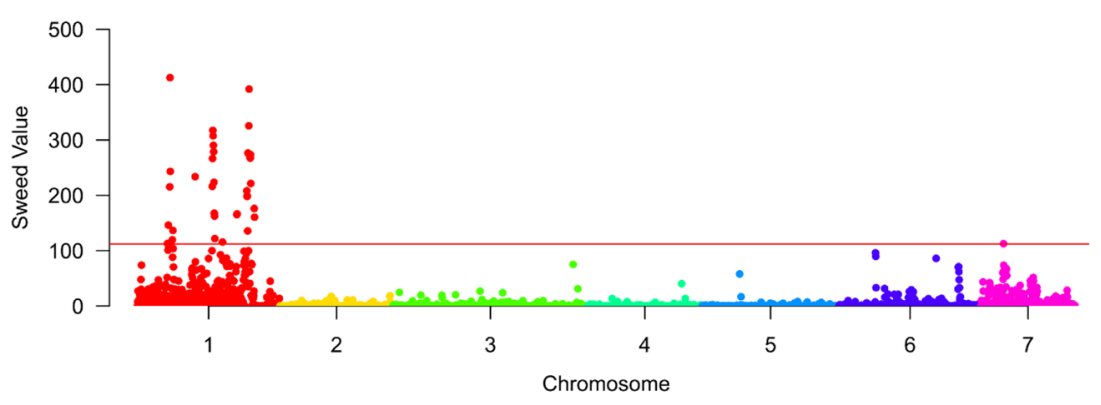

b

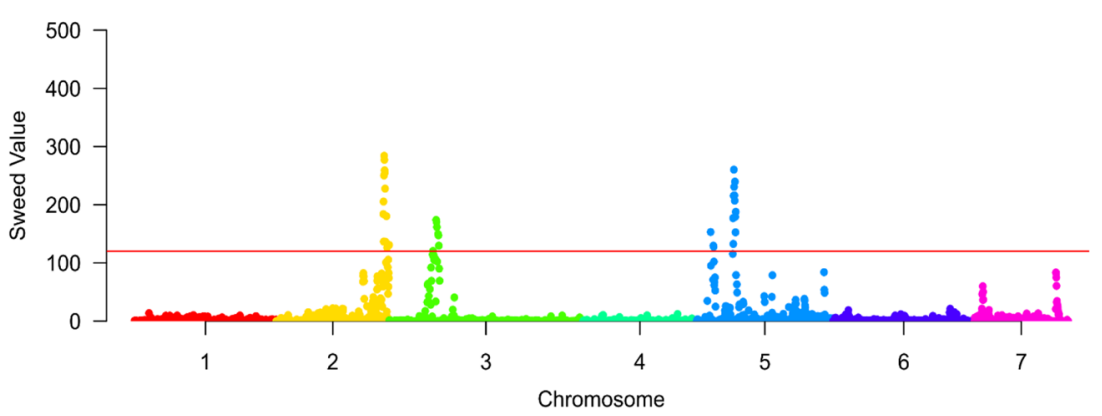

$\mathrm{c}$

d

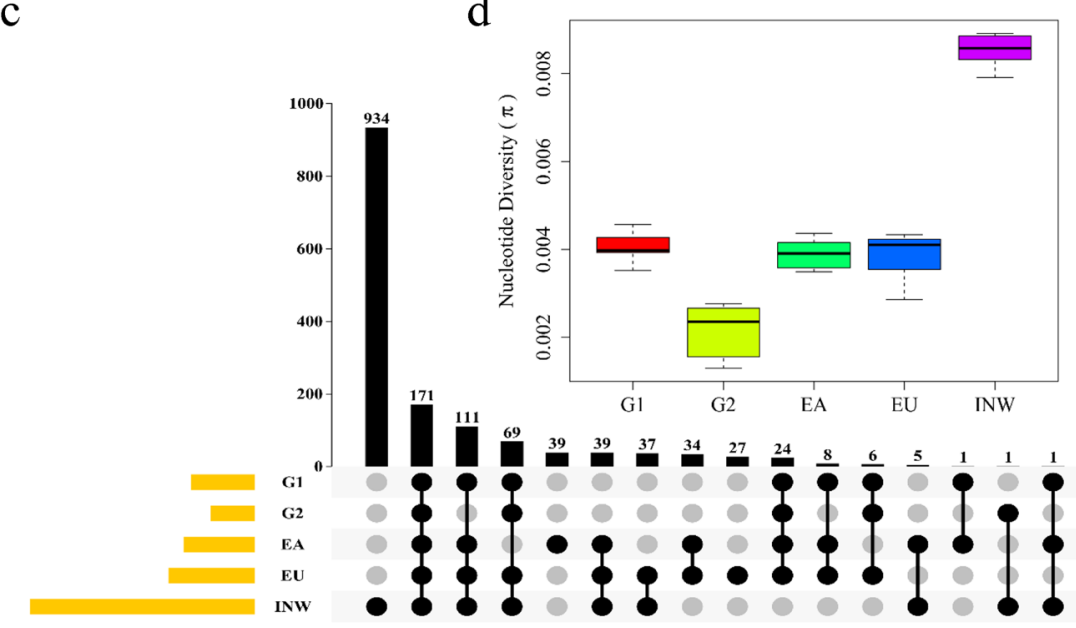

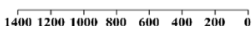

Fig. 3 Genes under artificial selection. a Selection signal in cucumber group 1. Red line indicates the top 1\%. b Selection signal in cucumber group 2. Red line indicates the top 1\%. c Venn diagram depicting unique and shared SNPs between the G1, G2, EA, EU, and INW populations in the powdery mildew resistance QTL interval Chr1:6815000-6858200. d Nucleotide diversity of 5 cucumber groups in the powdery mildew resistance QTL interval Chr1:6815000-6858200, as measured by the $\pi$ value

The different target selection regions in G1 and G2 might reflect breeders' preferences. In other words, most artificial selection results from the intentional breeding of target traits. However, selective breeding can also be unintentional $^{44}$. For example, in recent decades, we did not perform a disease resistance test to screen for resistant cucumber plants, but we unintentionally kept the healthy plants and removed disease-infected plants. To date, most plants in the G1 and G2 populations have performed well in the field and have not shown any disease infection. In this study, we surprisingly found that the powdery mildew resistance major-effect QTL $P m 1.1^{39}$ was located in a selection region. Then, we compared the SNPs in the Pm1.1 interval among the G1, G2, EA, EU, and INW groups (Fig. 3c) and found that the INW, EA, and EU groups had 934, 39, and 27 unique SNPs, respectively, while there were no unique SNPs in G1 or G2. In other words, the SNPs that existed in G1 and G2 were perhaps derived from the INW, EA and EU groups and fixed by artificial selection. Interestingly, G2 had a smaller number of SNPs as well as a lower nucleotide diversity $\left(\pi=2.14 \times 10^{-3}\right.$, $t$-test $\left.P<0.01\right)$ than the EA, EU, 
and INW groups (Fig. 3c, d), indicating that this interval was homozygous as a result of intensive selection and could be used to identify the causal region for powdery mildew resistance.

\section{Conclusions}

In this study, by using next-generation resequencing technology, we identified highly informative SNPs, which were used to study the genetic relations between cucumber germplasm accessions. Here, we reported 56 artificially selected cucumber inbred lines that exhibited various breeding preference phenotypes and could be divided into two groups. In addition, the two groups exhibited distinctive genetic structure due to their breeding history involving hybridization and selection, and they were significantly different from 115 previously reported cucumber lines. Phylogenetic inference with the TreeMix model revealed a breeding history in which G1 was improved based on the EA background and G2 was selected to change fruit traits mainly by crosses with the EU group. D-statistic values were consistent with positive selection in the cucumber populations, possibly related to domestication or selection on traits of interest. We also reported the possibility that selective breeding can be unintentional. Our results revealed the molecular basis of selection during cucumber development, and the 56 selected inbred lines could be good germplasm resources for cucumber breeding programs. For example, based on specific traits, each of the inbred lines could be used to develop populations, and the highly informative SNPs are of great relevance to cucumber breeders.

\section{Materials and methods}

\section{Sample collection and sequencing}

The cucumber population of 56 artificially selected inbred lines was collected, crossed and inbred at the Ren Laboratory of Vegetable Science, China Agricultural University. The population was grown in an experimental field in the Changping District, Beijing. Phenotyping was conducted in Changping (N 40 $13^{\prime}$, E $116^{\circ} 12^{\prime}$ ).

Genomic DNA was extracted as previously described ${ }^{15}$. At least $5 \mu \mathrm{g}$ of genomic DNA was prepared from a single plant of each accession for sequencing, and a library was constructed with an insert size of $\sim 300-350 \mathrm{bp}$ for all of the lines, following the manufacturer's instructions (Illumina HiSeq 2500, USA).

\section{Variant calling, filtering, and annotation}

The paired-end reads were aligned against the 9930 cucumber reference genome using the MEM algorithm implemented in BWA software ${ }^{45,46}$ after trimming lowquality reads by using Trimmomatic ${ }^{47}$. Picard tools (https://broadinstitute.github.io/picard/) was employed for the removal of PCR duplicates and realignment of indel regions. Generated files in Binary Alignment Map (BAM) format were used to call single nucleotide polymorphisms (SNPs) via the HaplotypeCaller function of Genome Analysis Toolkit (GATK, version 3.8) with default parameters ${ }^{48}$.

To obtain high-quality SNPs, a hard filtering step was performed by following the GATK Best Practices recommendations ${ }^{48}$. The genotype calls were then improved by imputing and phasing the polymorphism dataset with Beagle 4.1 software based on genotype likelihoods ${ }^{49}$. Finally, the effects of SNPs were predicted with SnpEff 4.3 software $^{50}$.

\section{Investigating population genetic structure}

We further thinned the SNP dataset by using the following rules: (1) core chromosomes (“--chr 1-7”); (2) minor allele frequency $>0.05$; (3) missing values $<10 \%$; (4) a Hardy-Weinberg equilibrium test (“--hwe 0.001"); and (5) pruning SNPs by “--indep-pairwise 5010 0.1”. Two parameters ("--pca" and "--distance-matrix") in PLINK software ${ }^{51}$ were used to calculate pairwise matrixes for the construction of a principal component analysis (PCA) plot and neighbor-joining tree, respectively. In addition, the ADMIXTURE (version 1.3.0) tool $^{52,53}$ was employed to estimate individual ancestry by setting the $K$-value from 2 to 7 . Nucleotide diversity measured by the $\pi$ value for each population was estimated by using VCFtools ${ }^{54}$ with a $50 \mathrm{~kb}$ window and $5 \mathrm{~kb}$ sliding steps. In addition, the decay of linkage disequilibrium (LD) with physical distance between SNPs was calculated and visualized by using PopLDdecay software ${ }^{55}$. Furthermore, TreeMix$1.12^{32}$ was used to model the genetic drift of genome-wide allele frequency data to infer population splitting and mixing. The standard errors of migration proportions were calculated by using the "-se" option. Migration edges (with the "-m" option) were added gradually from 0 to 5 .

\section{Identifying selective sweeps}

The difference in allele frequency between the G1 and G2 groups was estimated with BayeScan software (version $2.0)^{56}$, which decomposed locus-population $F_{\mathrm{ST}}$ coefficients into a population-specific component ( $\beta$ value) and a locus-specific component ( $\alpha$ value) using logistic regression. A positive $\alpha$ value suggests diversifying selection, whereas a negative value indicates balancing or purifying selection. A $q$-value $<0.05$, analogous to the $p$ value but used under multiple testing, was used to indicate significance. BayeScan software ${ }^{56}$ was run under the default parameters. To detect regions under complete selection within the population, the SweeD program was employed to calculate the composite likelihood ratio $(C L R)^{38,57}$, which identifies regions with significant deviations from the neutral site frequency spectrum (SFS). We set the top $1 \%$ as a significance threshold. Finally, the 
overlapping regions detected by $F_{\mathrm{ST}}$ and the CLR were identified as having signatures of selection. Gene Ontology (GO) annotations were assigned using AgriGO for cucumber (http://systemsbiology.cau.edu.cn).

\section{Acknowledgements}

This study was supported by the National Key Research and Development Program of China (2018YFD1000800), the National Natural Science Foundation of China (31830080), the Beijing Municipal Natural Science Foundation (6184043), and the Project of Beijing Agricultural Innovation Consortium (BAIC01). We are grateful to members of the Ren Laboratory for technical assistance and many discussions.

\section{Author details}

${ }^{1}$ Beijing Key Laboratory of Growth and Developmental Regulation for Protected Vegetable Crops, College of Horticulture, China Agricultural University, Beijing 100193, P. R. China. ${ }^{2}$ Centre for Research in Agricultural Genomics (CRAG), CSIC-IRTA-UAB-UB, Campus Universitat Autònoma de Barcelona, Bellaterra 08193, Spain. ${ }^{3}$ Department of Agronomy, College of Agriculture, Purdue University, West Lafayette, IN 47907, USA

\section{Author's contributions}

X.L. and H.R. proposed the project. B.L. and D.G. performed the data analysis and designed the manuscript structure, with the participation of J.H., H.R., X.L., B.L., X.Z., S.Y., S.X., and S.C. planted the population, scored the phenotypes and extracted DNA. B.L. wrote the paper, with help from D.G., X.L., and H.R.

\section{Conflict of interest}

The authors declare that they have no conflict of interest.

Supplementary Information accompanies this paper at (https://doi.org/ 10.1038/s41438-019-0209-4).

Received: 2 June 2019 Revised: 1 September 2019 Accepted: 13 October 2019

Published online: 15 November 2019

\section{References}

1. Huang, S. et al. The genome of the cucumber, Cucumis sativus L. Nat. Genet. 41, 1275 (2009).

2. Liu, B. et al. Silencing of the gibberellin receptor homolog, CsGID1a, affects locule formation in cucumber (Cucumis sativus) fruit. New Phytol. 210, 551-563 (2016).

3. Qi, J. et al. A genomic variation map provides insights into the genetic basis of cucumber domestication and diversity. Nat. Genet. 45, 1510 (2013).

4. Che, G. \& Zhang, X. Molecular basis of cucumber fruit domestication. Curr. Opin. Plant Biol. 47, 38-46 (2019).

5. Staub, J. E., Robbins, M. D. \& Wehner, T. C. in Vegetables I (eds Prohens, J. \& Nuez, F.) 241-282 (Springer, 2008).

6. Voss-Fels, K. P., Cooper, M. \& Hayes, B. J. Accelerating crop genetic gains with genomic selection. Theor. Appl. Genet. 132, 669-686 (2019).

7. Kozik, E. U. in Cucurbitaceae 2016, Xlth Eucarpia Meeting on Cucurbit Genetics \& Breeding, July 24-28, 2016, Warsaw, Poland. (Organizing Committee of Cucurbitaceae, 2016).

8. $\mathrm{Xu}, \mathrm{Y} . \&$ \& Crouch, J. H. Marker-assisted selection in plant breeding: from publications to practice. Crop Sci. 48, 391-407 (2008).

9. Fan, Z., Robbins, M. D. \& Staub, J. E. Population development by phenotypic selection with subsequent marker-assisted selection for line extraction in cucumber (Cucumis sativus L.). Theor. Appl. Genet. 112, 843-855 (2006).

10. Kooistra, E. Femaleness in breeding glasshouse cucumbers. Euphytica 16, 1-17 (1967).

11. Sitterly, W. R. Breeding for disease resistance in cucurbits. Annu. Rev. Phytopathol. 10, 471-490 (1972).

12. Yang, $X$. et al. Fine mapping of the uniform immature fruit color gene $u$ in cucumber (Cucumis sativus L.). Euphytica 196, 341-348 (2014).

13. Andeweg, J. \& De, BruynJ. Breeding of non-bitter cucumbers. Euphytica 8 , 13-20 (1959).
14. Hufford, M.-B. et al. Comparative population genomics of maize domestication and improvement. Nat. Genet. 44, 808 (2012).

15. Huang, X. et al. Genome-wide association studies of 14 agronomic traits in rice landraces. Nat. Genet. 42, 961 (2010).

16. Lam, H. M. et al. Resequencing of 31 wild and cultivated soybean genomes identifies patterns of genetic diversity and selection. Nat. Genet. 42, 1053 (2010).

17. Mace, E. S. et al. Whole-genome sequencing reveals untapped genetic potential in Africa's indigenous cereal crop sorghum. Nat. Commun. 4, 2320 (2013).

18. Duan, N. et al. Genome re-sequencing reveals the history of apple and supports a two-stage model for fruit enlargement. Nat. Commun. 8, 249 (2017).

19. Guo, S. et al. The draft genome of watermelon (Citrullus lanatus) and resequencing of 20 diverse accessions. Nat. Genet. 45, 51 (2013).

20. Pereira-Dias, L., Vilanova, S., Fita, A., Prohens, J. \& Rodríguez-Burruezo, A. Genetic diversity, population structure, and relationships in a collection of pepper (Capsicum spp.) landraces from the Spanish centre of diversity revealed by genotyping-by-sequencing (GBS). Hort. Res. 6, 54 (2019).

21. Huang, $X$. et al. Genome-wide association study of flowering time and grain yield traits in a worldwide collection of rice germplasm. Nat. Genet. $\mathbf{4 4}, 32$ (2012).

22. Wang, X. et al. The USDA cucumber (Cucumis sativus L.) collection: genetic diversity, population structure, genome-wide association studies, and core collection development. Hort. Res. 5, 64 (2018).

23. Zheng, Y. et al. Cucurbit Genomics Database (CuGenDB): a central portal for comparative and functional genomics of cucurbit crops. Nucleic Acids Res. 47, D1128-D1136 (2018).

24. Yang, X. et al. High-resolution mapping of the dull fruit skin gene $D$ in cucumber (Cucumis sativus L.). Mol. Breed. 33, 15-22 (2014).

25. Wang, Y.-L. et al. Identification and mapping of Tril, a homeodomain-leucine zipper gene involved in multicellular trichome initiation in Cucumis sativus. Theor. Appl. Genet. 129, 305-316 (2016)

26. Yang, $X$. et al. Tuberculate fruit gene Tu encodes a $\mathrm{C}_{2} \mathrm{H}_{2}$ zinc finger protein that is required for the warty fruit phenotype in cucumber (Cucumis sativus $\mathrm{L}$.) Plant J. 78, 1034-1046 (2014).

27. Yang, S. et al. A CsTu-TS 1 regulatory module promotes fruit tubercule formation in cucumber. Plant Biotechnol. J. 17, 289-301 (2018).

28. Guo, C. et al. Identification and mapping of ts (tender spines), a gene involved in soft spine development in Cucumis sativus. Theor. Appl. Genet. 131, 1-12 (2018).

29. Patterson, N., Price, A. L. \& Reich, D. Population structure and eigenanalysis. PLoS Genet. 2, e190 (2006).

30. Zhou, H., Alexander, D. \& Lange, K. A quasi-Newton acceleration for high-dimensional optimization algorithms. Stat. Comput. 21, 261-273 (2011).

31. Saitou, N. \& Nei, M. The neighbor-joining method: a new method for reconstructing phylogenetic trees. Mol. Biol. Evol. 4, 406-425 (1987).

32. Pickrell, J. K. \& Pritchard, J. K. Inference of population splits and mixtures from genome-wide allele frequency data. PLoS Genet. 8, e1002967 (2012).

33. Durand, E. Y., Patterson, N., Reich, D. \& Slatkin, M. Testing for ancient admixture between closely related populations. Mol. Biol. Evol. 28 2239-2252 (2011).

34. Hübner, S. et al. Sunflower pan-genome analysis shows that hybridization altered gene content and disease resistance. Nat. Plants 1, 54-62 (2018).

35. Bo, K, Ma, Z., Chen, J. \& Weng, Y. Molecular mapping reveals structural rearrangements and quantitative trait loci underlying traits with local adaptation in semi-wild Xishuangbanna cucumber (Cucumis sativus L. var. xishuangbannanesis Qi et Yuan). Theor. Appl. Genet. 128, 25-39 (2015).

36. Pan, Y. et al. QTL mapping of domestication and diversifying selection related traits in round-fruited semi-wild Xishuangbanna cucumber (Cucumis sativus L. var. xishuangbannanesis). Theor. Appl. Genet. 130, 1531-1548 (2017).

37. Liu, B. et al. A new grafted rootstock against root-knot nematode for cucumber, melon, and watermelon. Agron. Sustain Dev. 35, 251-259 (2015).

38. Pavlidis, P., Živković, D., Stamatakis, A. \& Alachiotis, N. SweeD: likelihood-based detection of selective sweeps in thousands of genomes. Mol. Biol. Evol. 30 2224-2234 (2013)

39. $\mathrm{Xu}, \mathrm{X}$. et al. Fine mapping of a dominantly inherited powdery mildew resistance major-effect QTL, Pm1. 1, in cucumber identifies a $41.1 \mathrm{~kb}$ region containing two tandemly arrayed cysteine-rich receptor-like protein kinase genes. Theor. Appl. Genet. 129, 507-516 (2016). 
40. Win, K. T., Vegas, J., Zhang, C., Song, K. \& Lee, S. QTL mapping for downy mildew resistance in cucumber via bulked segregant analysis using nextgeneration sequencing and conventional methods. Theor. Appl. Genet. 130, 199-211 (2017).

41. Lu, H. et al. QTL-seq identifies an early flowering QTL located near Flowering Locus T in cucumber. Theor. Appl. Genet. 127, 1491-1499 (2014).

42. Tan, J. et al. A novel allele of monoecious ( $m$ ) locus is responsible for elongated fruit shape and perfect flowers in cucumber (Cucumis sativus L.). Theor. Appl. Genet. 128, 2483-2493 (2015).

43. Shang, $Y$. et al. Biosynthesis, regulation, and domestication of bitterness in cucumber. Science 346, 1084-1088 (2014).

44. Purugganan, M. D. \& Fuller, D. Q. The nature of selection during plant domestication. Nature 457, 843 (2009).

45. Li, H. Aligning sequence reads, clone sequences and assembly contigs with BWA-MEM. arXiv Prepr. arXiv 1303, 3997 (2013).

46. Li, H. \& Durbin, R. Fast and accurate short read alignment with Burrows-Wheeler transform. Bioinformatics 25, 1754-1760 (2009).

47. Bolger, A. M., Lohse, M. \& Usadel, B. Trimmomatic: a flexible trimmer for Illumina sequence data. Bioinformatics 30, 2114-2120 (2014).

48. McKenna, A. et al. The Genome Analysis Toolkit: a MapReduce framework for analyzing next-generation DNA sequencing data. Genome Res. 9, 1297-1303 (2010).
49. Browning, B. L. \& Browning, S. R. Genotype imputation with millions of reference samples. Am. J. Hum. Genet. 98, 116-126 (2016).

50. Cingolani, P. et al. A program for annotating and predicting the effects of single nucleotide polymorphisms, SnpEff: SNPs in the genome of Drosophila melanogaster strain w1118; iso-2; iso-3. Fly 6, 80-92 (2012).

51. Purcell, S. et al. PLINK: a tool set for whole-genome association and population-based linkage analyses. Am. J. Hum. Genet. 81, 559-575 (2007).

52. Alexander, D. H. \& Lange, K. Enhancements to the ADMIXTURE algorithm for individual ancestry estimation. BMC Bioinform. 12, 246 (2011).

53. Alexander, D. H., Novembre, J. \& Lange, K. Fast model-based estimation of ancestry in unrelated individuals. Genome res. 9, 1655-1664 (2009).

54. Danecek, P. et al. The variant call format and VCFtools. Bioinformatics 27, 2156-2158 (2011)

55. Zhang, C, Dong, S.-S, Xu, J.-Y, He, W.-M. \& Yang, T-L. PopLDdecay: a fast and effective tool for linkage disequilibrium decay analysis based on variant call format files. Bioinformatics 10, 1786-1788 (2018).

56. Foll, M. \& Gaggiotti, O. E. A genome scan method to identify selected loci appropriate for both dominant and codominant markers: a Bayesian perspective. Genetics 2, 977-993 (2008).

57. Nielsen, R. et al. Genomic scans for selective sweeps using SNP data. Genome Res. 15, 1566-1575 (2005). 